Polymer Journal, Vol. 8, No. 5, pp 443-447 (1976)

\title{
Nuclear Magnetic Resonance of Oriented Poly(tetrahydrofuran)
}

\author{
Akihiro Tsutsumi, Kunio Hikichi, and Motozo Kaneko \\ Department of Polymer Science, Faculty of Science \\ Hokkaido University, Sapporo 060, Japan.
}

(Received April 23, 1976)

\begin{abstract}
Wide-line NMR studies of poly(tetrahydrofuran) (PTHF) have been made on drawn and rolled sheets in the range of temperature from -196 to $37^{\circ} \mathrm{C}$. The NMR spectra showed pronounced anisotropy with variation of the arrangement of the PTHF sheet in the applied magnetic field. The second moments of the NMR spectra for drawn and rolled sheets were well explained by the uniaxial and double orientations of the crystallites in the sheet. In the rolled sheet, the (100) plane of crystal, to which the zig-zag plane of the molecule is parallel, was found to be oriented preferentially parallel to the sheet plane. A reduction in the second moments of the crystalline regions with increasing temperature was interpreted in terms of the molecular motion in the crystal. A theoretical calculation showed that such a motion involves a rotational oscillation around the molecular axis.
\end{abstract}

KEY WORDS Poly(tetrahydrofuran) / NMR / Sheets / Second Moment / Molecular Motion / Crystal /

The molecular motion of poly(tetrahydrofuran) (PTHF) has been studied by dynamic mechanical, dielectric, and nuclear magnetic resonance (NMR) measurements. ${ }^{1-4}$ Willbourn ${ }^{1}$ and Wetton and Allen $^{2}$ have observed two mechanical relaxations at temperatures below $0^{\circ} \mathrm{C}$, which were denoted as $\beta$ - and $\gamma$-relaxations in descending temperature order. In the same temperature region, Wetton and Williams ${ }^{3}$ have found two dielectric relaxations. These relaxations have been interpreted in terms of segmental motion in the amorphous regions. We have carried out NMR measurements and found that PTHF molecules undergo molecular motion in the crystal. ${ }^{4}$ The details of such a motion, however, have not yet been clarified sufficiently.

In this work, we attempted to examine the molecular motion in the PTHF crystal in more detail. NMR measurements were carried out for drawn and rolled sheets of PTHF.

\section{EXPERIMENTAL}

PTHF with a viscosity average molecular weight of $5 \times 10^{4}$ was kindly supplied by Professor Miyake of Hokkaido University. Sheets of $0.5 \mathrm{~mm}$ in thickness were cast from concentrated benzene solution at a temperature of $20^{\circ} \mathrm{C}$. They were annealed at a temperature of $32^{\circ} \mathrm{C}$ for a week. Strips cut out from these sheets were drawn slowly in one direction at room temperature up to six times the original length. X-ray photographs of these drawn sheets showed a good orientation of the crystallites along the drawn direction. Some of the drawn sheets were rolled along the drawn direction at room temperature.

The drawn and rolled sheets were cut in a rectangular form and then packed parallel to one another in glass tubes for the NMR measurements.

The arrangement of samples in the magnetic field is shown in Figure 1. The direction of the magnetic field $H_{0}$ is specified by the angles $\omega$ and $\psi$ in the coordinate system $X Y Z$ fixed in the sheet. The $Y Z$ plane is parallel to the sheet

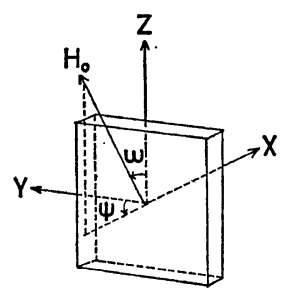

Figure 1. Direction of the NMR magnetic field with respect to the system $X Y Z$ fixed in the sheet (see text). 


\section{A. Tsutsumi, K. Hikichi, and M. Kaneko}

plane, and the $Z$ axis is parallel to the drawn or rolled directions.

Before the NMR measurements, the samples were evacuated for three days at $25^{\circ} \mathrm{C}$ to eliminate traces of benzene and other low molecular weight materials absorbed in the samples. The NMR apparatus was described in our previous paper. $^{4}$

\section{RESULTS AND DISCUSSION}

\section{Drawn PTHF}

Derivative absorption spectra for drawn PTHF are similar to those for other crystalline polymers, such as polyethylene and poly(oxymethylene). ${ }^{5}$ At lower temperatures the spectra consist of a single broad line, and at higher temperatures they become a broad line with a narrow line superimposed. Figure 2 shows representatives of derivative spectra for drawn PTHF at $\omega=0,45$, and $90^{\circ}$ at the indicated temperatures. The spectra show a pronounced anisotropy with variation of $\omega$, but not of $\psi$, indicating that the crystallites orient uniaxially along the drawn direction. A narrow line first appears in the spectrum of $\omega=0^{\circ}$ at $-70^{\circ} \mathrm{C}$, and is detected at all angles at $-50^{\circ} \mathrm{C}$. The distinction between the broad and narrow lines is considerably easier for spectra of $\omega=0^{\circ}$ than for those of other angles. The separtion between the maximum and the minimum of the broad line exhibits a pronounced angular dependence over the entire range of temperature studied.

The variation of the second moments of drawn PTHF against temperature is shown in Figure 3.

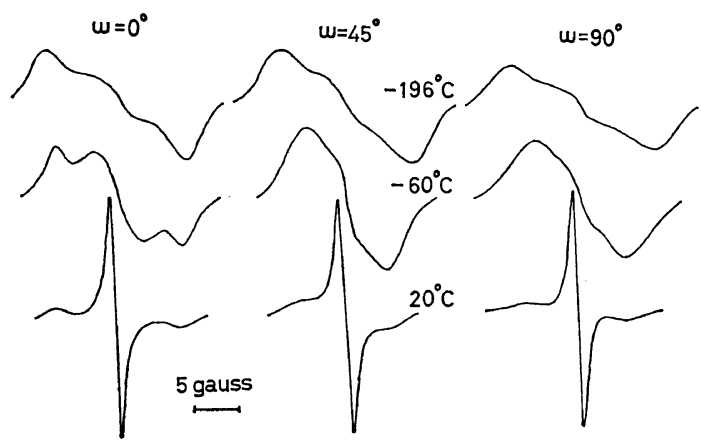

Figure 2. First derivatives of the proton resonance in drawn PTHF, obtained at alignment angles of $\omega=0,45$, and $90^{\circ}$ at the indicated temperatures.

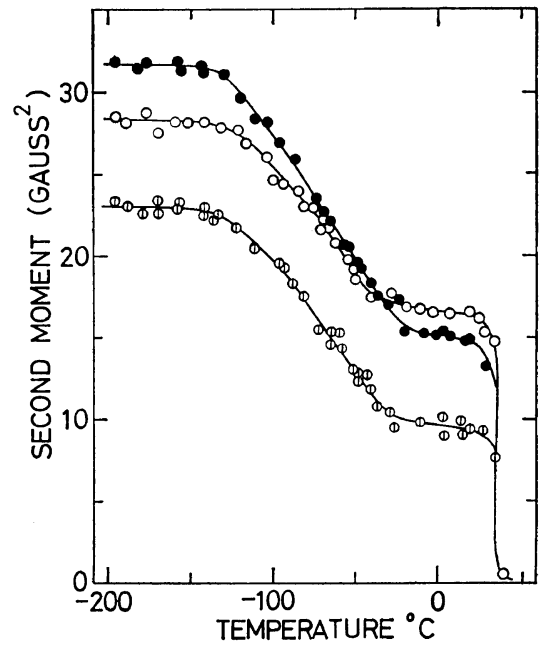

Figure 3. Plot of second moment versus temperature for drawn PTHF: $\bigcirc, \omega=0^{\circ}$; (1), $\omega=45^{\circ}$; , $\omega=90^{\circ}$.

There is an appreciable decrease in the second moment with increasing temperature between -196 and $20^{\circ} \mathrm{C}$. The decrease in second moment is more noticeable at $\omega=45$ and $90^{\circ}$ in this temperature range. The decrease at $\omega=0^{\circ}$, amounting to 11 gauss $^{2}$, is the smallest among the three angles. The second moment at $\omega=90^{\circ}$ is greatest at lower temperatures, but the one at $\omega=0^{\circ}$ is greatest at higher temperatures. The crossing point occurs at approximately $-35^{\circ} \mathrm{C}$. The second moment at $\omega=45^{\circ}$ is smaller than those at $\omega=0$ and $90^{\circ}$ at all temperatures studied.

It has been suggested that the two-phase model is adequate to describe the morphology of the crystalline polymers. The second moment of drawn PTHF may, then, be given by

$$
\left\langle\Delta H^{2}(\omega)\right\rangle_{\text {obs. }}=\left\langle\Delta H^{2}(\omega)\right\rangle_{\mathrm{c}}\left(1-X_{\mathrm{a}}\right)+\left\langle\Delta H^{2}(\omega)\right\rangle_{\mathrm{a}} X_{\mathrm{a}}
$$

where $\left\langle\Delta H^{2}(\omega)\right\rangle_{\mathrm{c}}$ and $\left\langle\Delta H^{2}(\omega)\right\rangle_{\mathrm{a}}$ are the second moments of the crystalline and amorphous regions, respectively, and $X_{\mathrm{a}}$ is the fraction of amorphous regions. This relation gives the second moment of the crystalline regions $\left\langle\Delta H^{2}(\omega)\right\rangle_{\mathrm{c}}$, if $\left\langle\Delta H^{2}(\omega)\right\rangle_{\mathrm{a}}$ and $X_{\mathrm{a}}$ are known.

It is known that the broad and narrow lines are contributed by the crystalline and amorphous regions, respectively, above the glass transition point $T_{\mathrm{g}}{ }^{6,7}$ The second moment of the amor- 
phous regions can be estimated from the narrow line above $T_{\mathrm{g}}$, if the two lines are well separated. For PTHF, this condition is well satisfied above $-35^{\circ} \mathrm{C}$, which is sufficiently above the glass transition point of $\mathrm{PTHF}-84^{\circ} \mathrm{C}^{2,3}$ At $20^{\circ} \mathrm{C}$, $\left\langle\Delta H^{2}(\omega)\right\rangle_{\mathrm{a}}$ was estimated to be 0.8 gauss $^{2}$ from the spectrum at $\omega=0^{\circ}$, which is very small compared with the second moment of the whole spectrum.

The unoriented sample which was well annealed at $32^{\circ} \mathrm{C}$ and the one quenched rapidly at $-196^{\circ} \mathrm{C}$ from the melt were found to have nearly the same value of the second moment, 28.5 gauss $^{2}$, at $-196^{\circ} \mathrm{C}$. This indicates that in randomly oriented PTHF the second moments contributed from the amorphous and crystalline regions are nearly the same at $-196^{\circ} \mathrm{C}$. For $\left\langle\Delta H^{2}(\omega)\right\rangle_{\mathrm{a}}$ at $-196^{\circ} \mathrm{C}$, we assumed this value and neglected the angular dependence in our calculation.

The fraction of amorphous regions, $X_{\mathrm{a}}$, may be given by the mobile fraction, ${ }^{8}$ i.e. the relative number of mobile protons, at temperatures sufficiently above $T_{\mathrm{g}}$, since all protons in amorphous regions contribute to the narrow line, due to the rapid segmental motion. Such a value was 0.43

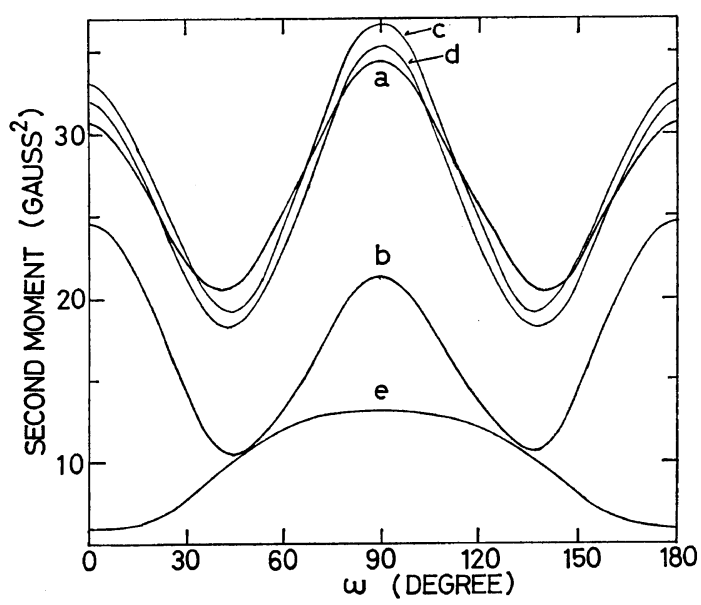

Figure 4. Second moment versus alignment angle. Curves $\mathrm{a}$ and $\mathrm{b}$ show the second moments of crystalline regions at -196 and $20^{\circ} \mathrm{C}$, respectively. Curves $\mathrm{c}$ and $\mathrm{d}$ show the calculated second moments for an uniaxial orientation at $-196^{\circ} \mathrm{C}$ and room temperature, respectively. Curve e shows the difference of the second moments between -196 and $20^{\circ} \mathrm{C}$. at $20^{\circ} \mathrm{C}$, where the mobile fraction-temperature curve was found to be leveling off.

The second moment- $\omega$ curves for the crystalline regions at -196 and $20^{\circ} \mathrm{C}$ are shown in Figure 4. A pronounced angular dependence of the second moment is observed at these temperatures. The angular dependence of the second moment decrease between these temperatures is also shown in Figure 4. The decrease is highly anisotropic. To explain these results, the theoretical second moment was calculated on the basis of uniaxial orientation of the crystallites.

The second moment of a fiber depends on the alignment angle as follow: ${ }^{9}$

$$
\left\langle\Delta H^{2}(\omega)\right\rangle_{\mathrm{c}}=A_{1} \sin ^{4} \omega+A_{2} \sin ^{2} \omega+A_{3}
$$

where $A_{1}, A_{2}$, and $A_{3}$ are constants which depend on the molecular geometry in the crystal and the degree of orientation of the crystallites along the fiber axis. It was assumed in this calculation that the crystals are uniaxially oriented completely and that the molecules are in the rigid state. The positions of protons in the molecule were determined from the X-ray results by Imada, et al. $^{10}$

The parameters $A_{1}, A_{2}$, and $A_{3}$ also depend on the crystal dimensions. The lattice constants of the PTHF crystal are reported to be $a=5.59$, $b=8.73$, and $c=12.07 \AA$ at room temperature. ${ }^{10}$ To determine the dimensions of the crystal lattice at low temperatures, the interplanar distances of the (130), (110), and (020) reflection planes were measured with changing temperature. It was found that $a$ increases remarkably with increasing temperature from $-130^{\circ} \mathrm{C}$, but the others do not change appreciably. At a temperature of $-196^{\circ} \mathrm{C}, a=5.39 \AA$ was obtained.

In Figure 4, the calculated second moment- $\omega$ curves are shown. At $-196^{\circ} \mathrm{C}$, the calculated curve explains fairly well the observed one, indicating that the PTHF crystals are well oriented along the drawn direction and that the molecules are in the rigid state. The reduction of the second moment due to the thermal expansion of the crystal lattice alone is very slight. The somewhat smaller values of the observed second moments at $20^{\circ} \mathrm{C}$, therefore, imply that molecules undergo molecular motion in the crystal.

For the evaluation of the theoretical second moment during motion, a rotational oscillation 


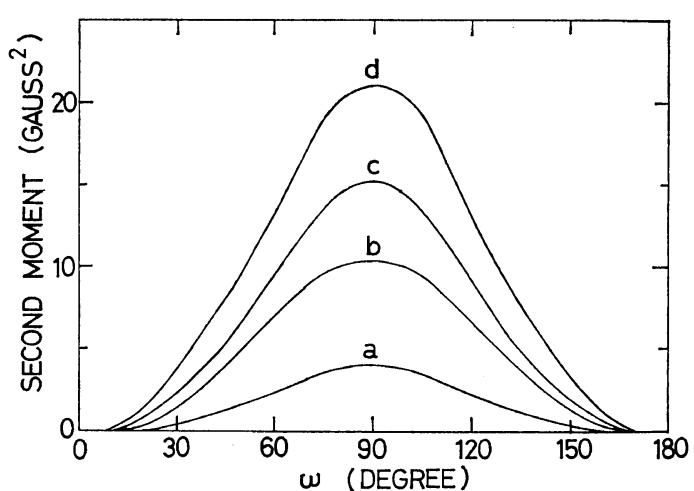

Figure 5. Decrease of the intramolecular second moment of a PTHF chain due to the rotational oscillation with amplitude $A,\left\langle\Delta H^{2}(\omega)\right\rangle_{\text {rigid chain }}$ $\left\langle\Delta H^{2}(\omega)\right\rangle_{\text {oscillation: }}$ a, $A=20^{\circ} ; \mathrm{b}, A=35^{\circ} ; \mathrm{c}, A=$ $50^{\circ} ; \mathrm{d}, A=\infty$.

of the molecule around the molecular axis was assumed. The evaluation was made only on the intramolecular contribution instead of the entire crystal lattice, because of the larger contribution of the former due to the factor $r^{-6}$, where $r$ is an inter-proton distance. The decrease in second moment from its rigid state value is shown in Figure 5 for various oscillation amplitudes.

The decrease in second moment is noticeable at $\omega=90^{\circ}$. This interprets qualitatively the anisotropy of the reduction in the observed second moment between -196 and $20^{\circ} \mathrm{C}$. It may be concluded that the rotational oscillation mode is one of the predominant mechanisms of the molecular motion in the PTHF crystal, as was found for other crystalline polymers. ${ }^{5,11-14}$

It can be seen in Figure 2 that the angular dependence of the reduction in second moment with increasing temperature is appreciable from $-130^{\circ} \mathrm{C}$. This implies that molecular motion in the crystal begins to occur far below room temperature. Furthermore, as described above, the thermal expansion of the crystal starts to occur from $-130^{\circ} \mathrm{C}$. These results suggest that there is a strong correlation between the thermal expansion of the crystal and the molecular motion in the crystal.

Rolled PTHF

Figures 6 and 7 show plots of the second moment of the rolled PTHF sheet at $-196^{\circ} \mathrm{C}$ against $\phi$ at $\omega=90^{\circ}$ and against $\omega$ at $\phi=90^{\circ}$,

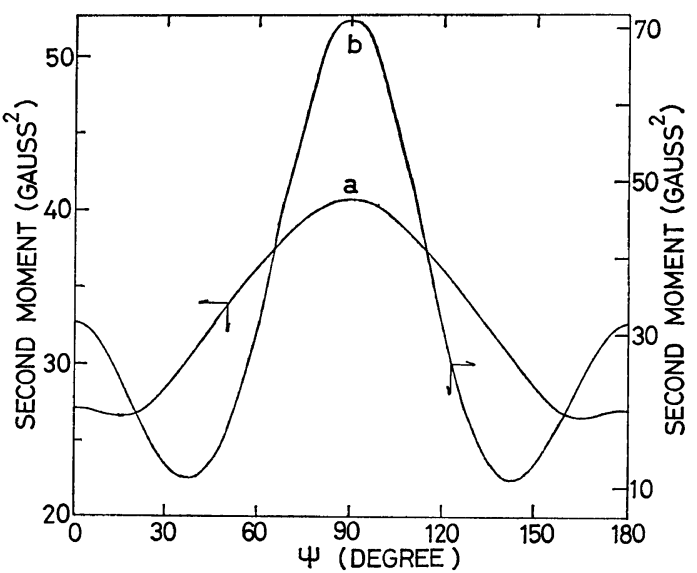

Figure 6. Second moment $v s$. $\phi$ for PTHF at $\omega=$ 90 : a, second moment of crystalline regions for rolled sheet at $-196^{\circ} \mathrm{C}$; b, calculated second moment for an ideal double orientation of crystallite in the sheet (see text).

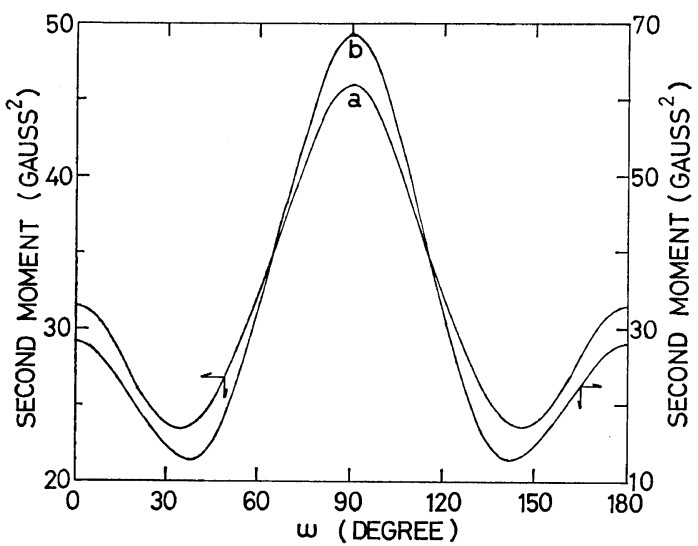

Figure 7. Second moment versus $\omega$ for PTHF at $\phi=90^{\circ}$ : a, second moment of crystalline regions for rolled sheet at $-196^{\circ} \mathrm{C} ; \mathrm{b}$, calculated second moment for an ideal double orientation of crystallite in the sheet (see text).

respectively. The pronounced angular dependences can be seen. These results are different from those for drawn PTHF, since the second moment depends not only on $\omega$, but also on $\psi$. To clarify these angular dependences, the observed second moments were compared with the theoretical ones.

We assumed a double orientation of the crystallites such that the (100) planes are parallel to the $Y Z$ plane, and the molecular axis ( $c$-axis) is parallel to the $Z$ axis, where the $X, Y$, and $Z$ 
axes are the coordinate system fixed in the sheet shown in Figure 1. The second moment formula as a function of the angles $\omega$ and $\phi$ is given by ${ }^{15}$

$$
\begin{aligned}
\left\langle\Delta H^{2}(\omega, \psi)\right\rangle_{\mathrm{c}}= & \sin ^{4} \omega\left(B_{1} \sin ^{4} \psi+B_{2} \sin ^{2} \psi+B_{3}\right) \\
& +\sin ^{2} \omega\left(C_{1} \sin ^{2} \psi+C_{2}\right)+D
\end{aligned}
$$

Here $B, C$, and $D$ are parameters independent of $\omega$ and $\psi$ and can be estimated from the structure of PTHF.

The calculated second moment curves are shown in Figures 6 and 7. The calculated curves show a rather strong angular dependence compared with the observed curves, owing to the assumption of ideal double orientation. The calculated second moment, however, explains qualitatively the angular dependences of the observed moments. It is concluded that in the rolled PTHF the crystal orients doubly: the (100) plane to which the zig-zag plane of the molecule is parallel orients preferentially parallel to the sheet plane, which is in agreement with the $\mathrm{X}$ ray results by Imada, et al. ${ }^{10}$

\section{SUMMARY AND CONCLUSION}

We measured NMR spectra of drawn and rolled sheets of PTHF over a wide range of temperature and at various arrangements in the magnetic field. It was confirmed by NMR measurements that in drawn and rolled sheets the crystallites orient uniaxially and doubly, respectively. In the rolled sheet, the (100) plane to which the zig-zag plane of the molecule is parallel was found to orient preferentially parallel to the sheet plane. The reduction of the second moment in the crystalline regions with increasing temperature is mainly due to the molecular motion within the crystal, since the effect of the thermal expansion is very slight. NMR results strongly suggest that such a motion involves the rotational oscillation around the molecular axis which begins to occur far below room temperature. It was suggested that there is a corre- lation between the onset of this motion and the thermal expansion of the crystal.

Acknowledgments. The author wish to express their gratitude to Professor Y. Miyake of Hokkaido University for kindly supplying the PTHF samples. We are indebted to Professor A. Chiba of Waseda University for valuable advice on the $\mathrm{X}$-ray diffraction measurements and helpful discussions. This work was supported by the Ministry of Education, Science, and Culture, Japan.

\section{REFERENCES}

1. A. H. Willbourn, Trans. Faraday Soc., 54, 717 (1958).

2. R. E. Wetton and G. Allen, Polymer, 7, 331 (1966).

3. R. E. Wetton and G. Williams, Trans. Faraday Soc., 61, 2132 (1965).

4. A. Tsutsumi, K. Hikichi, and M. Kaneko, Japan. J. Appl. Phys., 7, 577 (1968).

5. H. G. Olf and A. Peterlin, J. Appl. Phys., 35, 3108 (1964).

6. G. W. Wilson and G. E. Pake, J. Polym. Sci., 10, 5031 (1953).

7. D. W. McCall and E. W. Anderson, ibid., Part A-1, 1175 (1963).

8. W. P. Slichter, J. Appl. Phys., 32, 2339 (1961).

9. K. Yamagata and S. Hirota, Oyobutsuri (Japan), 29, 866 (1960).

10. K. Imada, T. Miyakawa, Y. Chatani, H. Tadokoro, and S. Murahashi, Macromol. Chem., 83, 113 (1965).

11. D. Hyndman and G. F. Origlio, J. Appl. Phys., 31, 1849 (1960).

12. K. Hikichi, T. Shibata, A. Tsutsumi, and J. Furuichi, J. Polym. Sci., Part A-2, 6, 653 (1968).

13. S. Iwayanagi and I. Sakurai, J. Macromol. Sci.Phys., B3(2), 259 (1969).

14. A. Chiba, A. Hasegawa, K. Hikichi, and J. Furuichi, J. Phys. Soc. Japan, 21, 1777 (1966).

15. H. G. Olf, J. Polym. Sci., Part A-2, 9, 1851 (1971). 